\title{
Virtual School Students' Reactions to Undertanding Tasks: The Case of Local Extrema Point
}

\author{
Shaker Rasslan ${ }^{1 *}$ (), Nedaa Shibli ${ }^{1}$, Amal Rasslan-Sharif ${ }^{2}$ (1)
}

\author{
${ }^{1} \mathrm{AL}-$ Qasemi Academic- College of Education, Baqa elgarbeia, 30100, ISRAEL \\ ${ }^{2}$ The Academic Arab College, Haifa. 32623, ISRAEL \\ *Corresponding Author: shaker1953@gmail.com
}

Citation: Rasslan, S., Shibli, N., \& Rasslan-Sharif, A. (2020). Virtual School Students' Reactions to Undertanding Tasks: The Case of Local Extrema Point. Contemporary Mathematics and Science Education, 1(2), ep20007. https://doi.org/10.30935/conmaths/8496

\begin{abstract}
The main aim this study is to examine the understanding of the concept of a local extreme point of differentiable function. Three research questions rose: (a) To what extent do students who study 5 mathematics units in virtual school understand the concept extremum point? (b) To what extent does the concept name (i.e. "extremum point") influence the mathematical understanding of the concept upon students who study 5 mathematics units in the virtual school? (c) To what extent do students who study 5 mathematics units in the virtual school understand the concept maximum point? To answer the research questions we used qualitative research methods based on semistructured interviews. The results of the study showed that: All the four interviewers know to find correctly the stationary points, but they don't distinguish between a stationary point and an Extremum point. All the interviewers mention that the two points are Extremum points instead of stationary points. We have to emphasize that mathematically, a stationary point not necessarily an extremum point. In addition, one of the interviewers confuses an extremum point and a maximum point because of the daily meaning of "Extremum" in Arabic languages. He also reveals Pseudo-Conceptual Behavior; he used sentences like "the tangent equal to 0" instead of the "slope of the tangent equal zero". It turns out also, that while three out of four interviewers determined the type of the extremum points by using the acceptable value table method correctly, the fourth determines the type of the extremum points by one point and by using the "odd function" characteristic, correctly. According to the requirement to find the extremum point the results showed that two of the four interviewers reveal pseudo-conceptual mode of thinking according to the maximum point concept. They use "slope at the extremum points equal 0". One of them even used concepts: increasing, decreasing, positive, negative meaninglessly, without clarifying who is positive (negative) or who is increasing (decreasing). One of the important but interesting results is the reaction of the interviewers to the definitions of the maximum point concept. The results showed that 3 students out of 4 gave correct mathematical definition, but; the $4^{\text {th }}$ student gave meaningless definition. We crossed his meaningless answer with his answers to the other questions during the interviews; it turned out that his answers to most of the questions revealed pseudo-conceptual mode of thinking.
\end{abstract}

Keywords: understanding tasks. extremum point. maximum (minimum) point

Received: 7 Jun. $2020 \bullet$ Accepted: 12 Jul. 2020

\section{INTRODUCTION}

Mathematical concepts vary across different languages, which may be related to how students understand the concepts. Literature dealing with the understanding of mathematical concepts and the mother tongue of students can be found in some examples: The concept "number" (Fuson \& Kwon, 1992), the concept "fraction" (Miura, et.al.,1999), the concept of "derivative" (Park, 2012) and the concept "solving $1^{\text {st }}$ order equation" (Rasslan \& Mashhari, 2020). One of our aims in this study is to examine the relation between language-specific terms for mathematical concepts and students' understanding of the extremum point concept.

Until this moment there are no translated instructional materials (IM) (textbooks, teachers' guides etc.) for 5-points (advanced level)
Arabic-speaking students in Israel (Rasslan \& Rasslan, 2017), It is written in Hebrew. This situation did not change in the IM at the virtual school either. One can find the following definition and tests for the type of extremum point (Schaum's Outlines, 2020):

\section{Definition: Local Extrema}

A function $f$ has a local maximum at $c$ if $f(c) \geq f(x)$ for all $x$ near $c$.

When we say near $c$, to be precise, we mean that there exists some open interval I around con which $f(c) \geq f(x)$ for all $x \in l$.

Similarly, $f$ has a local minimum at $c$ if $f(c) \leq(x)$ for all $x$ near $c$.

\section{Tests for Local Extrema Point}

In mathematics textbooks (Schaum's Outlines, 2020) one can find (as well as in the virtual school textbook) two tests to classify stationary points: 
1. Value Table for $\boldsymbol{f}^{\prime}(\boldsymbol{x})$ Test: Around the Stationary Points: If to the left of a stationary point $x_{o}, f^{\prime}(x)<0$ and on the right of $\left.x_{o}, f^{\prime}(x)\right\rangle 0$, then there is a local minimum on $x_{o}$. Similarly, for a local maximum point: If to the left of a stationary point $x_{o}$, $\left.f^{\prime}(x)\right\rangle 0$ and on the right of $x_{o}, f^{\prime}(x)\langle 0$, then there is a local maximum on $x_{0}$.

2. Second Derivative Test: given a differentiable function $f(x)$ with stationary point at $x_{o}$

a. If $\left.f^{\prime \prime}(x)\right\rangle 0$, the point $x=x_{o}$ is a local minimum.

b. If $f^{\prime \prime}(x)<0$, the point $x=x_{0}$ is a local maximum.

\section{THEORETICAL FRAMEWORK}

\section{Mathematical Understanding}

In this study, students' understanding of big ideas regarding the concept of derivative was investigated in the light of Skemp's (1976) theory on conceptualization of mathematical understanding: relational understanding (i.e., knowing both what to do and why) and instrumental understanding (i.e., knowing rules without reasons). A relational understanding of the concept of derivative demands making sense of certain relations among derivative. This requires not only knowing, but also being able to explain the role of these concepts in terms of what it means and why and how it is related to derivative. For instance, a student who understands the concept of derivative can explain how average rate of change approximates to the instantaneous rate of change, as well as how the slopes of secant lines approximate to the slope of tangent line by using the limit concept. Additionally, the student can explain why the instantaneous rate of change at a point is the same as the slope of the tangent at that point. On the other hand, an instrumental understanding of the derivative concept implies knowledge without making sense of what these concepts mean and how they are interrelated in the context of derivative.

\section{Concept Definition and the Concept Image}

Vinner (1991, p. 69) considered the concept definition and the concept image as two different "cells" in the cognitive structure and analyzed the introduction of a definition occurring in three possible scenarios:

1. The concept image changes to accommodate the definition.

2. The concept image remains as it is, the definition is forgotten or distorted.

3. The concept image and definition are both present but not linked together.

\section{Pseudo-Conceptual Thinking}

Following the last paragraph, the situation is more complex. Not only are other variants possible (e.g. distorted concept images produced by distorted definitions, Gray \& Pinto, 1995) but, more importantly, different types of connection between concept definition and concept image typically occur in a single individual. Vinner (1995) discusses how the concept image may change as a result of pseudo-conceptual thought, which seems on the surface to be conceptual behavior but lacks the reflective, analytic control procedures that characterize true conceptual thinking. It is possible for each individual to have different types of concept imagery with different links to the concept definition, including: informal imagery not deduced from a definition, which may be further subdivided into imagery consistent or inconsistent with the formal theory, distorted imagery produced from a distorted personal definition or by faulty reasoning from a correct definition, pseudo formal imagery, which may seem consistent with formal theory but is not ultimately deduced from the definition by formal reasoning, formal imagery deduced formally from the definition.

In practice it may be difficult to distinguish between these possibilities. If no definition has been given, only informal imagery is possible. (Erroneous informalities should not be classified as distorted imagery because the student has no formal definition to distort).

When a definition is given, informal ideas continue to be available and are essential to guide the individual (including the research mathematician) to formulate new ideas which can then be deduced formally. If a student remembers a (partially) correct definition, formal constructions become possible from the (correct part of the) definition, but quasi-formal and distorted imagery can also occur. The distinction between these requires investigation of the thought processes which led to their construction.

\section{Virtual School}

Virtual schooling (VS) was first employed in the mid-1990s and has become a common method of distance education used in K-12 jurisdictions. The most accepted definition of a virtual school is an entity approved by a state or governing body that offers courses through distance delivery - most commonly using the Internet (Barbour \& Reeves, 2009).

\section{LITERATURE REVIEW}

Following on from a previous study by the same authors (Rasslan \& Shibli, 2020) of the virtual school approach, it becomes clear that the understanding of the concept of the derivative is very limited. There was great difficulty in defining the concept as well as the relationship of the concept with its various representations: the symbolic, the geometric and the algebraic.

Fuson and Kwon (1992) showed the importance of number words for children to understand multi-digit numbers. In Korean, when they read a number, each digit in a number is said, and its value is named. For example, "11" is read as Ten one, in contrast to, eleven. Fuson and Kwon (1992) specified the difference between English and Korean number words as a possible linguistic reason why Korean children outperformed the U.S. children in multi-digit addition and subtraction. Similarly, Korean terms for fraction contain the part-whole concept, which is different from the corresponding English terms (Miura et al., 1999). For example, $1 / 2$ is read as sam-bun-ui-il, which is translated as "of three parts, one" (p. 3). It includes the concept that the whole is divided into three parts, unlike the term, "one third." Based on this observation, the authors conducted experiments with Korean and American first-grade students who had not learned fraction. During the experimental lessons, teachers explained how to read fractions and what they mean without emphasizing shaded and un-shaded parts in visual representations of fractions. OF three test administered in the middle and end of the first year, and the beginning of the second year, Korean students significantly outperformed American students in the last two tests, although their performance were not significantly different in the first test. Miura et al. (1999) argued that Korean terms, 


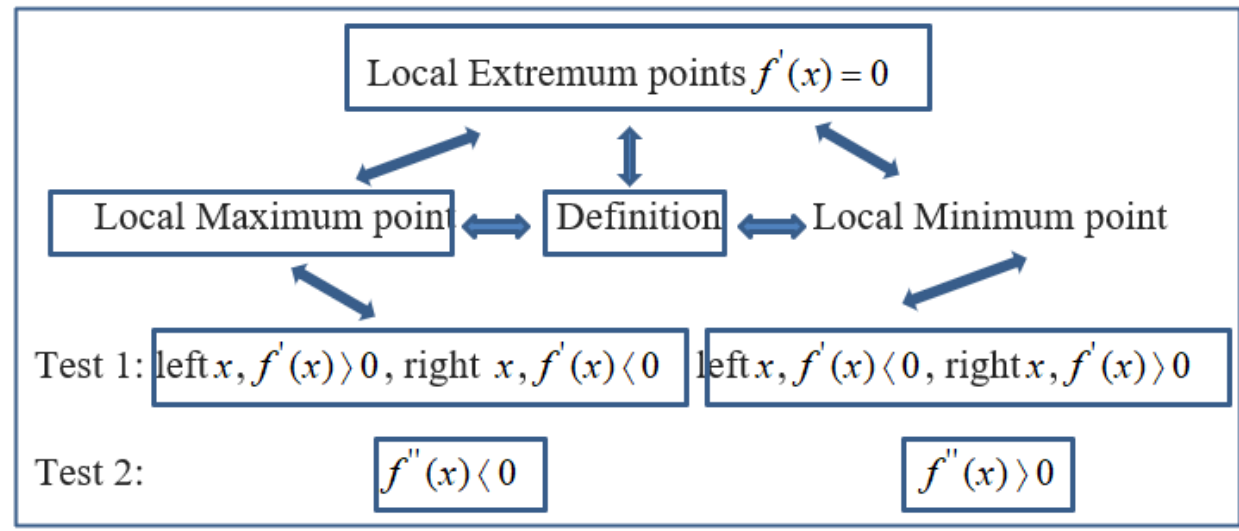

Figure 1. Relations between a local extrimum point concept and other related concepts

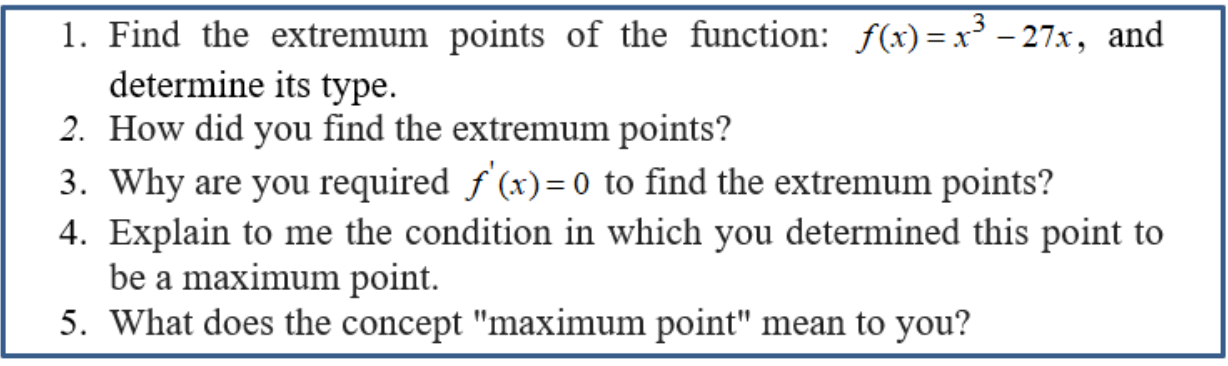

Figure 2. The questionnaire

which explicitly state the part-whole concept, may help children understand the simple fractions easier.

Another topic that may help our understanding of the students' thinking about the derivative would be the role that language plays in students' learning of mathematical concepts, For example, Park (2012) found different meanings in understanding the concept of the derivative, as revealed in the Korean and English languages.

Most existing studies have explored students' understanding about the derivative based on students' prior understanding and misconceptions of other concepts and their understanding of different representations of the derivative (Tall, 1986; Zandieh, 2000).

This study aims to examine secondary students' understanding of the related derivative concepts, such as a local extremum point, a local maximum (Minimum) point through VS: especially, the definitions, and the different tests to classify local extremum points as it is illustrated in Figure 1.

\section{Research Questions}

The research questions emerging from the literature review are:

1. To what extent do students who study 5 mathematics units in the virtual school understand the concept extremum point?

2. To what extent does the concept name (i.e. "extremum point") influence the mathematical understanding of the concept upon students who study 5 mathematics units in the virtual school?

3. To what extent do students who study 5 mathematics units in the virtual school understand the concept maximum point?

\section{METHODOLOGY}

\section{Sample}

Our sample included one $10^{\text {th }}$ grade class, in this class there were four out of ten students from the Arabic sector, studying mathematics at the 5-units level in VS.

\section{Semi-Structured Interviews}

The interviews are based on a questionnaire (Figure 2) consisting of five questions 1-5. The questionnaire was compiled by the authors in Arabic. The purpose of Question 1 was to reveal students' ability to find and determine the type of the extremum points. The aim of question 2 was to examine the students' ability to explain how they find the extremum points. The aim of question 3 was to examine the students ability to explain the condition $f^{\prime}(x)=0$ to find the extremum points. The aim of question 4 was to examine the students' understanding of the tests for maximum (minimum) points and finally the aim of question 5 was to reveal the definitions of the concept local maximum point. We settled on the maximum point because the explanation could be similar to the minimum point.

\section{Method}

The second author is the one who conducted the students' mother tongue interviews- Arabic. The interviews were conducted for each student individually. Each student received a blank solutions copybook from the interviewer so that they would write their answers in the notebook.

At the end of the interviews, the copybooks were collected by the interviewer for documentation and analysis. An interview with each student lasted about an hour. 


\section{RESULTS}

In the interviews analysis, we used qualitative analysis methods similar to analysis methods used in other studies (Tsamir, Rasslan \& Dreyfus, 2006). For the convenience of the reader, in the analysis, we will present the question, and then answer all four interviewees on which we will mark in S1-S4. After presenting the students 'answers, we summarize the four interviewees' analysis of the same question.

\section{Understanding the Concept "Extrenum Point"}

Question 1: Find the extremum points of the function: $f(x)=x^{3}-27 x$, and determine its type

S1: The first thing is to derive the function. Since we know that the extemum points of the function are present when they are at the highest point, the tangent is equal to zero. "Ok, here are two points $(x=-3),(x=3)$. These are the extremum points."

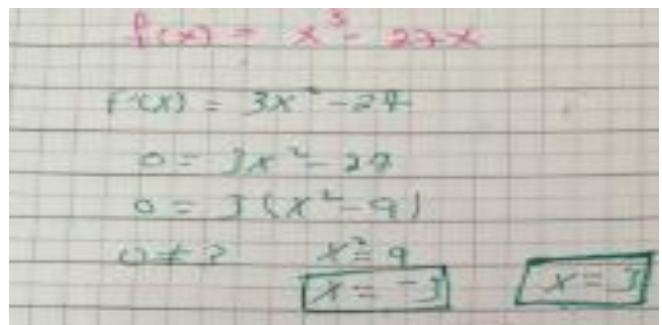

To determine the type of extremum points, we choose values around the extremum points, and then we calculate the $y$ values... $f(-10)=$ $-730, f(-3)=54, f(0)=0, f(3)=-54, f(10)=730$...here we can examine whether the function is odd or not: $f(-x)=-x^{3}+27 x=$ $-\left(x^{3}-27 x\right)=-f(x)$ for this reason $(-3,54)$ is maximum and $(3,-54)$ is minimum.

S2: From the derivative equal to 0 , the points were taken out. $x=3, x=-3$.

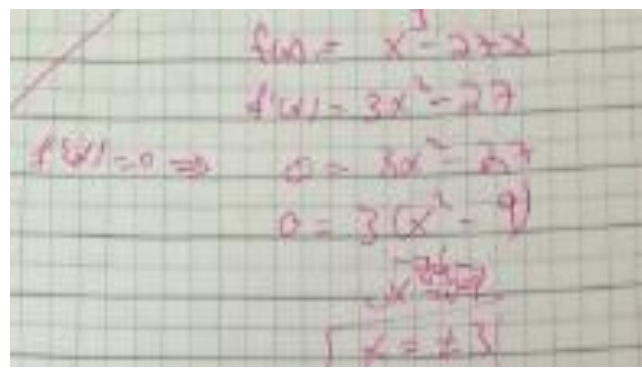

Specify the type according to the table... I substitute in the derivative to know its slope. For example: If it is negative...positive, it means that it is minimum, if it is positive ... negative it means that it is maximum, and if it is positive and positive or negative and negative it is stationary.

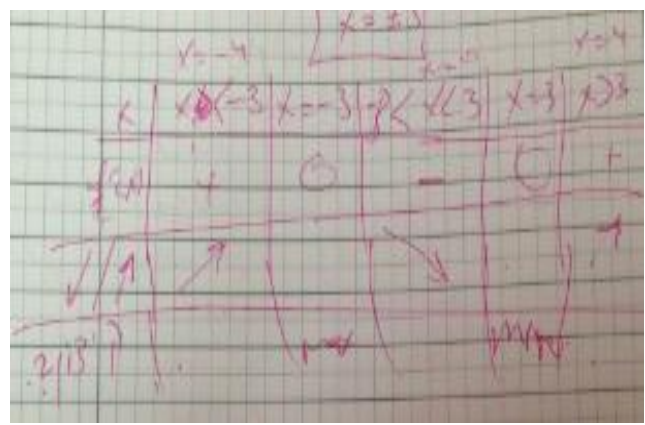

S3: $\quad F(X)=X^{3}-27 X \Rightarrow f^{\prime}(x)=3 x^{2}-27 \Rightarrow 0=3 x^{2}-$ $27 \Rightarrow x^{2}-9=0 \Rightarrow x= \pm 3$.

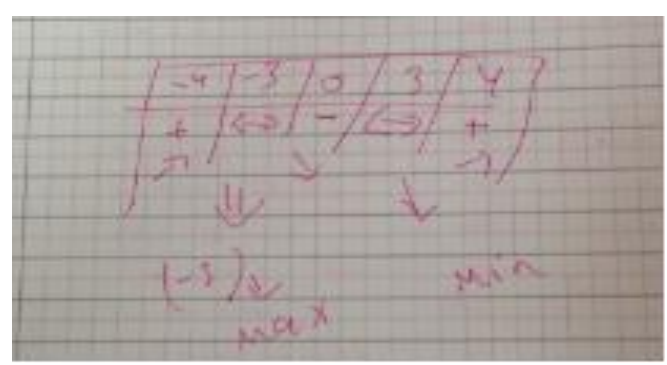

S4: I derivative the function and let the derivative equal zero. $f^{\prime}(x)=3 x^{2}-27=0 \Rightarrow x^{2}=9 \Rightarrow x= \pm 3$. These are critical points.

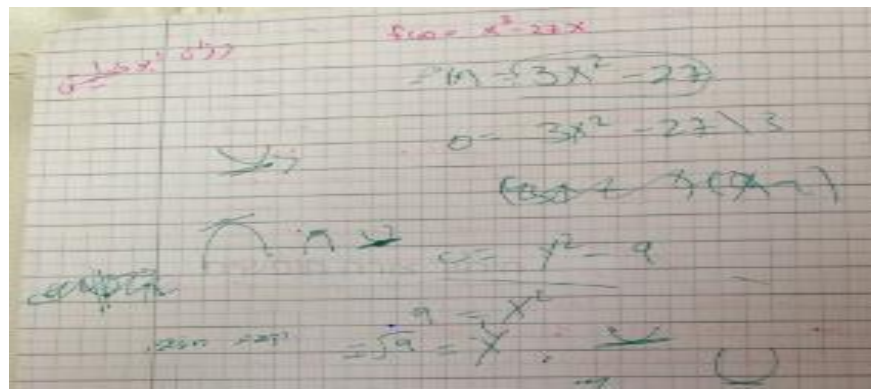

Before the maximum point it should be increasing and after it it should be decreasing. For this reason, before -3 it is increasing and after it is decreasing, so it is maximum.

From the above interviews, all the interviewers S1-S4 know to find correctly the stationary points. But $\mathrm{S} 1$ confuses an extremum point and a maximum point because of the daily meaning of "Extremum" in Arabic languages (the highest), he also reveals Pseudo-Conceptual Behavior; he used sentences like "the tangent equal to 0".

It turns out also, that while three out of four interviewers (S2-S4) determined the type of the extremum points by using the acceptable value table method correctly, S1detemined the type of the extremum points by one point and the "odd function" characteristic, correctly.

Question 2: How did you find the extremum points?

S1: I find the extremum points according to the slope of the function at the points where the derivative is equal to 0 , meaning the slope equals zero, and we output the slope according to the derivation of the function."

S2: I derived the function, I equaled the derivative to 0 , and I solved the equation.

S3: I find the derivative and equal it to 0.

S4: To find the extremum points, we must find the derivative...we equal the derivative to 0 .

From the above interviews, all the interviewers S1-S4 know to find the stationary points correctly, but, no one of the interviewers S1-S4 distinguished between stationary point and extremum point.

Question 3: Why are you required $f^{\prime}(x)=0$ to find the extremum points?

S1: Because the slope at this point is equal to 0....means that it is the point that is not increasing or that after it is decreasing or before it is negative or after it is positive...

S2: Because if you draw a tangent at the extremum point, the derivative equal to 0 , i.e. the tangent is parallel to $x$-axis, i.e. the slope of the tangent is 0 .

S3: The slope of the function at the extremum point of the function. It is equal to 0.... it is parallel to the $x$-axis and is equal to zero.

S4: The slope of the extremum point always equal 0 . There is no slope for the extremum point, for this reason we put it equal it to 0 . 
From the above, it turns out that two of the interviewers (S1, S4) reveal Pseudo-Conceptual Behavior, they use "slope at the extremum points equal 0". S1 even used mathematical concepts: increasing, decreasing, positive, negative meaninglessly, without clarifying who is positive (negative) or who is increasing (descending). S2, S3 reactions are correct.

\section{Understanding the Concept a Maximum Point}

Question 4: Explain to me the condition in which you determined this point to be a maximum point

S1: Since -3 is the highest point in the neighborhood (compensation $x$ is close to -3.

S2: According to the one before and the next one after, for example, if it is negative positive it is minimum, and if it is positive negative it is maximum.

S3: It means, before it positive and after is negative.

S4: Before -3 it is increasing and after it is decreasing, so it is maximum. It turns out that except from S1, all the other interviewers S2-S4 revealed pseudo-conceptual of thinking according maximum point. They use words like: positive negative or increasing decreasing without any reminder who is positive/negative or who is increasing/decreasing.

\section{Question 5: What does the concept "maximum point" mean to you?}

S1: It has the highest value of $y$ in the neighborhood around it.

S2: I mean, it is from among those around it, it is the largest, and it means the largest value, the greatest value from among those around it.

S3: I mean, it is from among those around it, it is the largest one. The largest value is from among those around it. Anything before or after it is below. It means, its $y$ is the highest. It means, it is transition point from positive to negative.

S4: It means that the domain stop at it means that it is the end of the domain.

It turns out that the interviewers S1-S3 defined correctly the concept "a maximum point": S1 by using the classical definition "it is the highest point". S4 gave a meaningless definition.

\section{DISCUSSION}

The main aim of the present study was to examine the understanding of the concept of a local extreme point of differentiable function; this is in continuation of (Rasslan \& Sheble, 2020; Park, 2012) concerned with the understanding of the derivative concept. To answer the research questions we used qualitative research methods based on semi-structured interviews.

The first research question was: To what extent do students who study 5 mathematics units in the virtual school understand the concept extremum point?

\section{The results of the study showed that:}

All the interviewers know to find correctly the stationary points, but they don't distinguish between a stationary point and an Extremum point. All the interviewers mention that the two points are Extremum points instead of stationary points. We have to emphasize that mathematically, a stationary point not necessarily an extremum point. This result is new.
The second research question was: To what extent does the concept name (i.e. "extremum point") influence the mathematical understanding of the concept upon students who study 5 mathematics units in the virtual school?

The result showed that one student (out of four) of the interviewers confuses an extremum point and a maximum point because of the daily meaning of "Extremum" in Arabic languages (the highest). This result is similar to the findings of (Park, 2012). He found different meanings in understanding the concept of the derivative, as revealed in the Korean and English languages; in our case between Hebrew and Arabic languages. This result is similar also to the findings of (Rasslan \& Mashare, 2020). They found that students don't understand the concept "solution of the $1^{\text {st }}$ order equations" as a result of using English instructional mathematics material in English language instead of Arabic language.

He also reveals Pseudo-Conceptual Behavior; he used sentences like "the tangent equal to 0 " instead of the "slope of the tangent equal zero". This result is similar to finding in other studies (Vinner, 1995; Rasslan \& Vinner, 1997, 1998) concerning other concepts.

It turns out also, that while three out of four interviewers determined the type of the extremum points by using the acceptable value table method correctly, the fourth student determines the type of the extremum points by one point and by using the "odd function" characteristic, correctly. This result new too.

According to the requirement $f^{\prime}(x)=0$ to find the extremum point the results showed that two of our four interviewers reveal pseudo-conceptual mode of thinking according to the maximum point concept. They use "slope at the extremum points equal 0". One of them even used mathematical concepts: increasing, decreasing, positive, negative meaninglessly, without clarifying who is positive (negative) or who is increasing (descending). These results are new and similar to othe findings mentioned in other studies (Vinner, 1995; Rasslan \& Vinner, 1997, 1998) concerning other concepts.

One of the important but interesting results is the reaction of the interviewers to the definitions of the maximum point concept (research Question 3). The results showed that 3 students out of 4 gave correct mathematical definition, but; the $4^{\text {th }}$ student gave meaningless definition to the concept. We crossed his meaningless answer with his answers to the other questions during the interviews; it turned out that his answers to most of the questions revealed pseudo-conceptual mode of thinking. This result also similar to other findings of other studies: (Vinner, 1995; Rasslan \& Vinner, 1997, 1998) concerning other concepts (odd/even funcction, increasing/decreasing function). These profiles of students don't know the concept definition of the concept, instead; he developed strange concept images (Vinner, 1991).

\section{CONCLUSION}

In this study we examine the understanding of the concept "a local extremum point" of a given differentiable function who studied mathematics in special environment; called "Virtual School". The results showed that the extent of understanding the concept "a local extremum point" is weak; the relationship between the concept of an exrimum point with other related concepts; maximum (minimum) point or the condition $f^{\prime}(x)=0$ are quite loose, even they studied mathematics in advanced level. They must learn mathematics during different learning 
environment and during their mother tongue. To use username and password may increases the capital of their creators, but not their understanding of the concept.

\section{REFERENCES}

Barbour, M., \& Reeves, T., (2009). The Reality of Virtual Schools: A review of the literature. Computers \& Education, 52(2), 402-416. https://doi.org/10.1016/j.compedu.2008.09.009

Fuson, K., \& Kown, Y., (1992). Korean Children's Understanding Multidigit Addition and Substraction. Child development, 63(2), 491506. https://doi.org/10.2307/1131494

Miura. I., Okamoto, Y., Vlahovic-Stetic, V., Kim, C., \& Han, J. (1999) Language supports for children's understanding of numerical fractions: cross-national comparison. Journal of Experimental Child Psychology, 74, 356-265. https://doi.org/10.1006/jecp.1999.2519

Park J. (2012). Students' Understanding of the Derivative- Literature Review of English and Korean Publications. Journal of the Korean School Mathematics Society, 15(2), 331-348. Retrieved from https://www.koreascience.or.kr/article/JAKO201223052005068. page

Pinto, M. M. F., \& Gray, E. (1995). Difficulties Teaching Mathematical Analysis to Non specialists. Proceedings of PME 19, Recife, Brazil, 11, 18-25.

Rasslan S., \& Mashhari, M., (2019). The Impact of Using Microsoft Math Software on Understanding Mathematical Central Concepts Upon Non-English Speaking Students- the Case Of The " $1{ }^{\text {st }}$ Degree Equations" Concept. $5^{\text {th }}$ International Contemporary Educational Research Congress. Theory and Practice in Education. July 25-27, 2019. Frankfurt University of Applied Sciences. Germany.

Rasslan, S., \& Shibli, N., (2020). Students' Understanding of the Derivative Concept by “The Virtual School”. (to be published).
Rasslan, S., \& Vinner, S. (1998). Images and definitions for the concept of increasing/decreasing function. In A. Oliview \& K. Newstead (Eds.), Proceedings of the 22nd conference of the international group for the psychology of mathematics education (Vol. 4, pp. 33-40), Jul 12-17. Stellenbosch: PME

Rasslan, S., \& Vinner. S. (1997). Images and Definitions for the Concept of Even / Odd Function. In Proceeding of the PME 21st 42 Conference of the International Group for the Psychology of Mathematics Education (vol. 4, pp. 41-48). University of Helsinki, Lahti, Finland.

Schaum's Outlines, (2020). Calculus, sixth Edition, Chap. 14. NAGWA. https://www.nagwa.com/en/courses/342179296797/

Skemp, R. R. (1976). Relational Understanding and Instrumental Understanding. Mathematics Teaching, 77, 20-26.

Tall, D. (1986). Construction the concept image of a tangent. In Proceeding of the eleventh conference of the International Group for the Psychology of Mathematics Education (vol. 3, pp. 69-75), Montreal.

Tsamir, P., Rasslan, S. \& Dreyfus T., (2006). Prospective teachers reactions to Right-or-Wrong tasks: The case of derivatives of absolute value functions. The Journal of Mathematical Behavior, 25(3), 240-251. https://doi.org/10.1016/j.jmathb.2006.09.001

Vinner, S. (1991). Role of definitions in the teaching and learning of mathematics. In D. O. Tall (ed.), Advanced Mathematical Thinking (pp. 65-81). Dordrecht: Kluwer. https://doi.org/10.1007/0-30647203-1_5

Vinner, S. (1995). Teaching mathematics as an educational task teachers' views about some aspects of their professional lives. Proceedings of the 19th international conference for the psychology of mathematics education. Recife, (III), 328-335. https://files.eric.ed.gov/fulltext/ED411136.pdf

Zandieh, M. (2000) A theoretical Framework for analyzing students understanding of the concept of derivative. CBMS Issues in Mathematics Education, 8, 103-127. https://doi.org/10.1090/ cbmath/008/06 